\title{
FREQUENCY SHIFTS IN STIMULATED RAMAN SCATTERING
}

\author{
W. ZINTH and W. KAISER \\ Physik Department der Technischen Universität München, \\ München, Fed. Rep. Germany
}

Received 3 December 1979

\begin{abstract}
The nonresonant contributions to the nonlinear susceptibility $\chi^{(3)}$ produce a frequency chirp during stimulated Raman scattering. In the case of transient stimulated Raman scattering, the spectrum of the generated Stokes pulse is found at higher frequencies than expected from spontaneous Raman data. The frequency difference can be calculated from the theory of stimulated Raman scattering.
\end{abstract}

It has been shown in numerous papers that an intense electromagnetic wave of frequency $\omega_{\mathrm{L}}$, travelling through a Raman active medium, is able to generate a strong Stokes shifted collinear wave at frequency $\omega_{\mathrm{S}}$. In most experimental investigations the Stokes shift $\omega_{0}=\omega_{\mathrm{L}}-\omega_{\mathrm{S}}$ of the stimulated Raman process was found to be equal to the Stokes shift known from spontaneous Raman measurements [1]. In contradiction, there exists a number of papers where deviations in the Stokes shifts between stimulated and spontaneous Raman scattering were reported [2].

In this note we present a theoretical and experimental study of the stimulated Raman process taking into account the self-phase modulation of the laser and Stokes wave. It will be shown that under quasistationary conditions the stimulated Stokes emission has the same Stokes shift as spontaneous Raman scattering. For the transient stimulated Raman process the situation is different. Theory predicts and experiments confirm a smaller Stokes shift, i.e. a higher Stokes frequency. For clarity, we consider here a specific case, Raman scattering of a molecular vibration in liquids. The theoretical discussion is quite general being applicable to other Raman type process as well.

The nonlinear response of the molecules is represented by the nonlinear polarisation $P^{\mathrm{NL}}$, which may be separated into two parts: A resonant contribution $P_{\mathrm{R}}^{\mathrm{NL}}$ due to the molecular transition of interest with a Raman susceptibility $\partial \alpha / \partial q$ and a nonresonant part
$P$ NR due to the electronic states of the molecules [3].

$P^{\mathrm{NL}}=P_{\mathrm{R}}^{\mathrm{NL}}+P_{\mathrm{NR}}^{\mathrm{NL}}=N \frac{\partial \alpha}{\partial q}\langle q\rangle E+\chi^{\mathrm{NR}(3)} E E E$.

$N$ is the number density of the molecules and $\langle q\rangle$ denotes the expectation value of the normal mode operator $q$ for the transition. For simplicity we make two assumptions: (i) A highly polarized Raman line is investigated, i.e. $\partial \alpha / \partial q$ is a scalar. (ii) $\chi^{\mathrm{NR}(3)}$ is taken to be time independent since the time constants of the electronic contributions are approximately $10^{-14}$ s. The slower material response is described by the equation of motion $[4,5]$ :

$\frac{\partial^{2}}{\partial t^{2}}\langle q\rangle+\frac{2}{T_{2}} \frac{\partial}{\partial t}\langle q\rangle+\omega_{0}^{2}\langle q\rangle=\frac{1-2 n^{\prime}}{2 m} \frac{\partial \alpha}{\partial q} E^{2}$.

$\omega_{0}$ and $m$ represent the transition frequency and the reduced mass, respectively, $T_{2}$ the dephasing time and $n^{\prime}$ is the occupation probability of the first excited vibrational level. For the system considered here we have a small population, i.e. $n^{\prime} \ll 1$.

For the light fields and the coherent material excitation, we make the ansatz of plane waves propagating in the $x$-direction:

$E=\frac{1}{2}\left\{\sum_{j} E_{j} \exp \left(-\mathrm{i} \omega_{j} t+\mathrm{i} k_{j} x+\mathrm{i} \phi_{j}\right)+\mathrm{cc}\right\}$

and

$\langle q\rangle=\frac{1}{2} \mathrm{i}\left\{Q \exp \left(-\mathrm{i} \omega_{\mathrm{q}} t+\mathrm{i} k_{\mathrm{q}} x+\mathrm{i} \phi_{\mathrm{q}}\right)+\mathrm{cc}\right\}$. 
The index $j=\mathrm{S}$ and $\mathrm{L}$ indicates the Stokes and laser wave, respectively. Energy and wave vector conservation is ensured by the equations:

$\omega_{\mathrm{q}}=\omega_{\mathrm{L}}-\omega_{\mathrm{S}}$ and $k_{\mathrm{q}}=k_{\mathrm{L}}-k_{\mathrm{S}}$.

Introducing eqs. (1) to (4) into the wave equation and considering slowly varying fields one obtains $[3,5]$ :

$$
\begin{aligned}
& \frac{\partial}{\partial t} Q+i Q \frac{\partial \phi_{\mathrm{q}}}{\partial t}+\frac{1}{T_{2}} Q+\frac{\mathrm{i}\left(\omega_{0}^{2}-\omega_{\mathrm{q}}^{2}\right)}{2 \omega_{\mathrm{q}}} Q \\
& =\frac{1}{4 m \omega_{\mathrm{q}}} \frac{\partial \alpha}{\partial q} E_{\mathrm{L}} E_{\mathrm{S}} \exp \left\{\mathrm{i}\left(\phi_{\mathrm{L}}-\phi_{\mathrm{S}}-\phi_{\mathrm{q}}\right)\right\},
\end{aligned}
$$

$$
\begin{aligned}
& \frac{\partial E_{\mathrm{S}}}{\partial x^{\prime}}+\mathrm{i} E_{\mathrm{S}} \frac{\partial \phi_{\mathrm{S}}}{\partial x^{\prime}}=\frac{\pi \omega_{\mathrm{S}}}{c n_{\mathrm{S}}}\left\{\frac{\partial \alpha}{\partial q} N E_{\mathrm{L}} Q \exp \left[\mathrm{i}\left(\phi_{\mathrm{L}}-\phi_{\mathrm{S}}-\phi_{\mathrm{q}}\right)\right]\right. \\
& \left.+\mathrm{i} 6 \chi_{\mathrm{SS}}^{\mathrm{NR}(3)} E_{\mathrm{S}}^{3}+\mathrm{i} 6 \chi_{\mathrm{LS}}^{\mathrm{NR}(3)} E_{\mathrm{L}}^{2} E_{\mathrm{S}}\right)
\end{aligned}
$$$$
\frac{\partial E_{\mathrm{L}}}{\partial x^{\prime}}+\mathrm{i} E_{\mathrm{L}} \frac{\partial \phi_{\mathrm{L}}}{\partial x^{\prime}}=\frac{\pi \omega_{\mathrm{L}}}{c n_{\mathrm{L}}}\left\{-\frac{\partial \alpha}{\partial q} N E_{\mathrm{Q}} Q \exp \left[-\mathrm{i}\left(\phi_{\mathrm{L}}-\phi_{\mathrm{S}}-\phi_{\mathrm{q}}\right)\right]\right.
$$$$
\left.+\mathrm{i} 6 \chi_{\mathrm{SL}}^{\mathrm{NR}(3)} E_{\mathrm{S}}^{2} E_{\mathrm{L}}+\mathrm{i} 6 \chi_{\mathrm{LL}}^{\mathrm{NR}(3)} E_{\mathrm{L}}^{3}\right\} \text {. }
$$

In eqs. (5) to (7) we introduced the retarded time frame $x^{\prime}=x$ and $t^{\prime}=t-x / v$. Group velocity $(v)$ dispersion should be neglected here. $n_{\mathrm{L}}, n_{\mathrm{S}}$ are the refractive indices at the laser and Stokes frequencies $\omega_{\mathrm{I}}$ and $\omega_{\mathrm{S}} . c$ is the velocity of light. The factors $\chi_{i j}^{\mathrm{NR}(3)}$ are the important phase changes generated by the intense light fields. $\chi_{i j}^{\mathrm{NR}(3)}$ with $i=j$ determines the self-phase modulation of the laser and Stokes fields (selfaction) while $i \neq j$ gives the effect of the field $E_{i}$ on the field $E_{j}$. Far from electronic resonances the different factors, $\chi_{i j}^{N R(3)}$, are of similar magnitude. When dispersion is neglected the theory of nonlinear susceptibilities gives: $\chi_{i i}^{\mathrm{NR}(3)}=0.5 x_{i j}^{\mathrm{NR}(3)}$ for $i \neq j$ [6]. For small conversion of laser into Stokes light, the underlined terms in eqs. (6) and (7) containing $E_{\mathrm{S}}^{3}, E_{\mathrm{S}}^{2}$, and $E_{\mathrm{S}} Q$ may be omitted.

We begin the discussion of eqs. (5) to (7) with the quasi-stationary situation where the interaction time is long compared to the dephasing time $T_{2}$. In this case the time derivative of $Q$ in eq. (5) may be neglected. Substitution of $Q$ into eq. (6) allows to solve the equation for the Stokes field. One readily finds the well-known exponential amplification of the Stokes intensity $I_{\mathrm{S}}(x)=I_{\mathrm{S}}(0) \exp \left(g I_{\mathrm{L}} x\right)$. At the resonance frequency where $\omega_{0}=\omega_{q}=\omega_{\mathrm{L}}-\omega_{\mathrm{S}}$, the gain coefficient has the form $g=\left(4 \pi^{2} \omega_{\mathrm{S}} N T_{2}(\partial \alpha / \partial q)^{2}\right) /$ $\left(n_{\mathrm{S}} n_{\mathrm{L}} m c^{2} \omega_{0}\right)$. The gain is not influenced by the nonresonant susceptibility of the medium.

In fig. 1a we present a calculated Stokes pulse (broken line) generated by a gaussian shaped laser pulse (solid line). Both pulses are normalized to their peak values. As a consequence of the strong experimental amplification $I_{\mathrm{S}}(x) / I_{\mathrm{S}}(0)=7 \times 10^{10}$, the stimulated Stokes pulse is generated only during a short period near the maximum of the laser pulse $(t=0)$. The duration of the Stokes pulse is one fifth of the laser pulse duration for the amplification considered here (conversion efficiency of $\eta=10^{-2}$ ).

The time dependent phases $\phi_{\mathrm{L}}(t)$ and $\phi_{\mathrm{S}}(t)$ are deduced from the imaginary parts of eqs. (6) and (7). The shape of the laser pulse (i.e. $E_{\mathbf{L}}(t)$ ) and the magnitude of the nonlinear coefficients $\chi_{\mathrm{LL}}^{\mathrm{NR}(3)}$ and

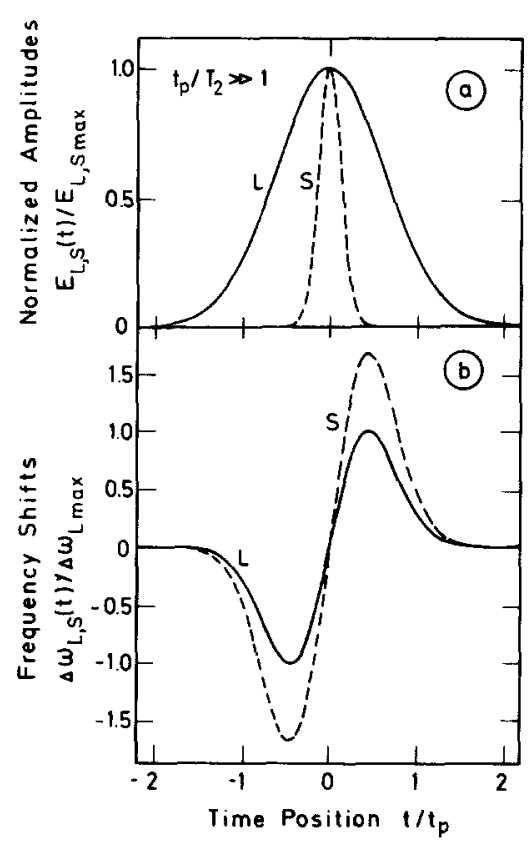

Fig. 1. Quasistationary stimulated Raman scattering including a nonresonant nonlinear susceptibility $\chi^{\mathrm{NR(3)}}$ calculated for a a gaussian laser pulse. (a) The normalized amplitudes of the laser field (solid line) and the Stokes field (broken line). (b) The shifts of the momentary frequencies of the laser (solid line) and the Stokes pulse (broken line). The time dependence of the frequency shift is determined by the shape of the laser pulse and the value of the nonlinear susceptibilities $\times \mathrm{NR}_{\mathrm{LL}}^{\mathrm{N}(3)}=$ $0.5 \times \mathrm{NR}(3)$. 
$\chi_{\mathrm{LS}}^{\mathrm{NR}(3)}$ determine $\phi_{\mathrm{L}}(t)$ and $\phi_{\mathrm{S}}(t)$ at the center of the Raman band, $\omega_{S}=\omega_{L}-\omega_{0}$. The Stokes field of smaller intensity does not affect the phases. The momentary frequencies of the laser and Stokes waves is given by the relation: $\omega_{\mathrm{L}, S}(t)=\omega_{\mathrm{L}, S}(0)-\partial \phi_{\mathrm{L}, S} / \partial t$. In fig. $1 \mathrm{~b}$ we show the frequency shifts of laser and Stokes radiation: $\Delta \omega_{\mathrm{L}, S}(t)=\omega_{\mathrm{L}, S}(t)-\omega_{\mathrm{LS}}(0)=$ $-\partial \phi_{L S S} / \partial t$. The time dependence of the laser frequency has the familiar pattern resulting from the gaussian laser pulse. The value of $\Delta \omega_{\mathrm{Lmax}}$ is estimated from the relation $[3,5]$ :

$$
\begin{aligned}
& \frac{\Delta \omega_{\mathrm{L} \max }}{2 \pi c}=2 \times 10^{-13} \frac{\omega_{\mathrm{L}} n_{2}}{2 \pi c n_{\mathrm{L}}} \frac{I_{\mathrm{L}} l}{t_{\mathrm{p}}} \\
& =2 \times 10^{-13} \frac{\widetilde{\nu}_{\mathrm{L}}}{n_{\mathrm{L}}} \frac{n_{2} G}{t_{\mathrm{p}} g} .
\end{aligned}
$$

In the right term, $\widetilde{v}_{\mathrm{L}}$ is the laser frequency in $\mathrm{cm}^{-1}$, $n_{2}=\left(12 \pi / n_{\mathrm{L}}\right) \chi_{\mathrm{LL}}^{\mathrm{NR}(3)}$ the nonlinearity of the medium in esu, $G=g I_{\mathrm{L}} l$ the Raman gain, $g$ the Raman gain coefficient in $\mathrm{cm} /$ watt, $t_{\mathrm{p}}$ the pulse duration in seconds, and $l$ the cell length in $\mathrm{cm}$. For the following practical parameters, $\widetilde{\nu}_{\mathrm{L}}=19000 \mathrm{~cm}^{-1}, n_{2}=1.6 \times 10^{-12} \mathrm{esu}$, $G=25, n_{\mathrm{L}}=1.5$, and $g=10^{-9} \mathrm{~cm} / \mathrm{W}$, one estimates a small frequency shift of $\Delta \omega_{L \max } / 2 \pi c<1 \mathrm{~cm}^{-1}$ for a pulse duration of $t_{\mathrm{p}}>0.1 \mathrm{~ns}$. In fig. $1 \mathrm{~b}$ the shift of the Stokes frequency (broken line) is 1.67 times larger than the shift of the laser when $\chi_{\mathrm{LL}}^{\mathrm{NR}(3)} \simeq 0.5$ $\chi_{\mathrm{LS}}^{\mathrm{NR}(3)}$ and $\widetilde{\nu}_{\mathrm{S}} \simeq 0.83 \widetilde{\nu}_{\mathrm{L}}$ is assumed in the calculation. Of interest is the question here how the frequency shifts influence the spectrum of the Stokes emission. In a Raman generator the amplification starts from noise with Stokes components $\omega_{\mathrm{S}} \neq \omega_{\mathrm{L}}-\omega_{0}$ over the homogeneous linewidth $\Delta \omega_{\mathrm{Sp}}=2 / T_{2}$. The large amplification of $G=g I_{\mathrm{L}} x \sim 25$ leads to a gain narrowing of approximately a factor of six [3]. In fig. $1 \mathrm{~b}$ the shift $\Delta \omega_{\mathrm{S}}(t)$ changes sign at the peak of the Stokes pulse $(t=0)$ and $E_{\mathrm{S}}$ as well as $\left|\Delta \omega_{\mathrm{S}}(t)\right|$ are symmetrical to $t=0$. Thus the center of the Stokes spectrum does not change. The frequency shift $\Delta \omega_{S}$ and the resulting spectral broadening is inversely proportional to $t_{\mathrm{p}}$. For quasistationary Raman scattering with $t_{\mathrm{p}} \gg$ $T_{2}$ (or $\Delta \omega_{\mathrm{Sp}} \gg 1 / t_{\mathrm{p}}$ ) the broadening due to the nonresonant term $\chi^{\mathrm{NR}(3)}$ is negligible.

Different results are obtained for transient stimulated Stokes scattering, when $t_{\mathrm{p}} \simeq T_{2}$. One readily sees from eq. (8) that the larger transient gain of $G \simeq 100$ and the shorter pulses of several picoseconds give fre-

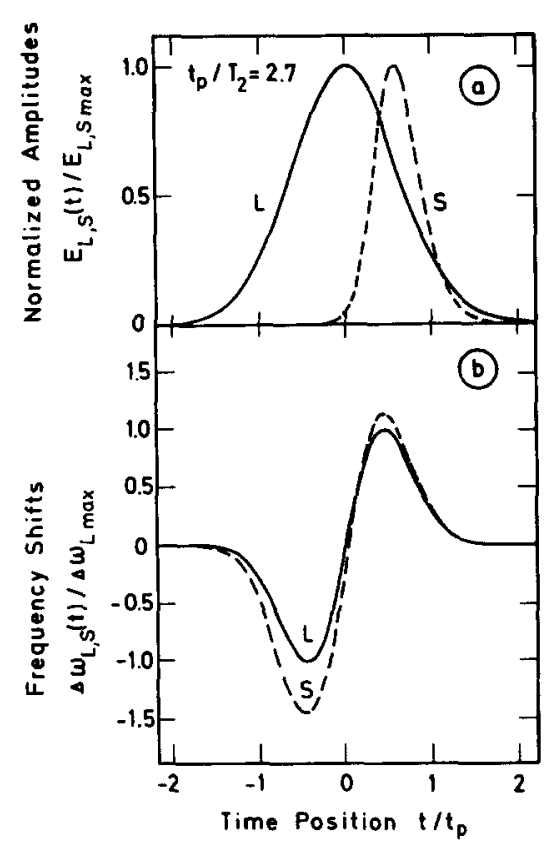

Fig. 2. Transient stimulated Raman scattering including a nonresonant nonlinear susceptibility $\chi^{\mathrm{NR}(3)}$. The calculations are carried out for a gaussian laser pulse with a duration of $t_{\mathrm{p}}=$ $2.7 T_{2}$, and for nonresonant susceptibilities $\times \mathrm{NR}^{(3)}=0.5$

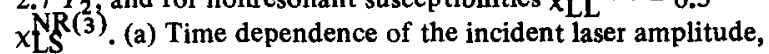
$\mathrm{L}$, (solid line) and of the generated Stokes amplitude, $S$, (broken line), (b) Shift of the momentary frequencies of the laser (solid curve) and the Stokes pulse (broken curve). Near the maximum of the Stokes amplitude the laser and Stokes pulses have a similar frequency shift.

quency shifts $\Delta \widetilde{\nu}_{L}=\Delta \omega_{L} / 2 \pi c$ of approximately 40 $\mathrm{cm}^{-1}$. For the transient case eqs. (5) to (7) have to be solved numerically. As discussed previously, the slow growth of the material excitation leads to a delayed peak of the Stokes emission [5,8]. The calculations of figs. 2 and 3 are made for the following material parameters: Gain $g=8 \times 10^{-10} \mathrm{~cm} / \mathrm{W}$, dephasing time $T_{2}=2.6 \mathrm{ps}$, nonlinear refractive index $n_{2}=1.6 \times 10^{-13} \mathrm{esu}$, and for the system parameters: Gaussian shaped laser pulse of duration $t_{\mathrm{p}}=2.7 \times T_{2}$, conversion efficiency of Stokes to laser energy $\eta_{s}=$ $10^{-2}$, and nonresonant susceptibilities $\chi_{\mathbf{L L}}^{\mathrm{NR}(3)}=0.5$ $\chi_{\mathrm{LS}}^{\mathrm{NR}(3)}$.

Fig. 2a shows the (normalized) laser input pulse and the (normalized) Stokes pulse. The peak of the latter is delayed by approximately $t_{\mathrm{D}}=t_{\mathrm{p}} / 2$. Calculations show that values of $t_{\mathrm{D}}$ and of the peak of the Stokes pulse are not affected by the small nonreso- 


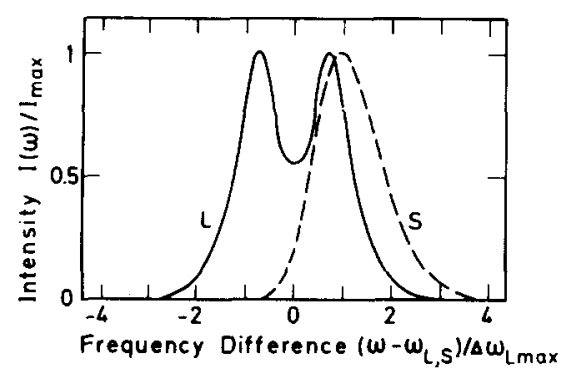

Fig. 3. Normalized spectrum of the transmitted laser pulse (L) and of the generated Stokes pulse (S, broken line) calculated for the parameters used in fig. 2 . The laser spectrum is centered around $\omega_{L}$ showing self-phase modulation. The Stokes spectrum is shifted to higher frequencies. $\omega_{S}$ represents the Stokes frequency of spontaneous Raman scattering.

nant term $\chi^{\mathrm{NR}(3)}$ considered here. For larger values of $\chi^{\mathrm{NR}(3)}$ and taking into account the color dispersion of the medium, one expects a decrease of the transient Stokes amplification $[8,9]$.

In fig. $2 b$ the frequency shifts of laser and Stokes radiation are depicted as a function of time. The normalized frequency shift of the transmitted laser pulse (solid line) is the same for the transient and for the quasistationary situation. The Stokes field (broken line), however, shows less frequency shift in the transient case. This fact is particularly evident at the time of the peak of the Stokes field Inspection of eqs. (5) to (7) shows that the difference between laser and Stokes shifts decreases with growing material excitation (more accurately, with growing ratio of material excitation $Q$ to driving force $E_{\mathrm{S}} E_{\mathrm{L}}$ ). In the transient case the material excitation reaches its maximum value at the end of the laser pulse. As a result, at $t=t_{\mathrm{p}}$ the laser and Stokes shifts have equal values and the molecular vibration oscillates with its resonance frequency.

The time dependence of the laser and Stokes pulse (fig. 2a) and the change of the momentary frequencies (fig. 2b) leads to distinct spectra of the laser and Stokes radiation. In fig. 3 the (normalized) spectral intensities are plotted versus frequency differences where $\omega_{L}$ represents the carrier frequency of the input laser pulse and $\omega_{S}=\omega_{L}-\omega_{0}$ the Stokes frequency without self-phase modulation. The spectrum of the transmitted laser pulse shows two maxima resulting from the self-phase modulation by the nonresonant susceptibility $\chi_{\mathrm{LL}}^{\mathrm{NR}(3)}$. The radiation is distributed symmetrically around the center frequency
$\omega_{\mathbf{L}}$ of the incoming laser pulse. The spectrum of the Stokes pulse (broken line) is not centered around the position $\omega_{\mathrm{S}}$ of the spontaneous Raman spectrum. Most of the Stokes radiation is generated at a delayed time when a large frequency shift occurs (see fig. 2). Thus the Stokes spectrum is moved by $\Delta \omega_{\mathrm{S}} \simeq$ $\Delta \omega_{\mathrm{Lmax}}$. Since $\Delta \omega_{\mathrm{Lmax}}$ is positive at the trailing part of the laser pulse the whole spectrum is shifted to higher frequencies.

In our experiments we investigated transient stimulated Raman scattering with picosecond laser pulses. We start with a single chirp-free infrared pulse from a passively mode-locked Nd:glass laser system [10]. The pulse is frequency doubled in a KDP crystal to $\widetilde{\nu}_{\mathrm{L}}=$ $18990 \mathrm{~cm}^{-1}$. The input pulse to the system has nearly gaussian shape and a duration of $t_{\mathrm{p}} \simeq 6.5 \mathrm{ps}$. A lens of $f=60 \mathrm{~cm}$ first forms a convergent beam; beyond the focus in the divergent beam the Raman cell is located. Self-focussing within the cell of $2 \mathrm{~cm}$ length is not observed and is not expected from numerical estimates. Care was taken to image only the central part of the transmitted laser beam [10] onto the slit of a $2 \mathrm{~m}$ grating spectrograph. In this way, the self-phase modulation of the intense part of the beam is clearly observed. The spectra of the laser and Stokes pulses are monitored by an optical multichannel analyser. The spectral resolution of the system is $0.2 \mathrm{~cm}^{-1}$ and absolute frequency scale is known with an accuracy of $0.4 \mathrm{~cm}^{-1}$.

We report experimental data of liquid $\mathrm{CH}_{3} \mathrm{CCl}_{3}$. Transient stimulated Raman scattering in $\mathrm{CH}_{3} \mathrm{CCl}_{3}$ is observed from the $\mathrm{CH}_{3}$ stretching mode with a spontaneous Raman shift of $2939 \mathrm{~cm}^{-1}$. Three experimental spectra are presented in fig. 4. Fig. 4a shows the narrow incoming laser pulse at the laser frequency of $18990 \mathrm{~cm}^{-1}$. We know from simultaneous measurements of the temporal and spectral properties that the incident pulse has no frequency chirp [10]. Quite different is the spectrum of the laser pulse at the end of the Raman cell (fig. 4b). The spectrum with two maxima is typical for a pulse with small self-phase modulation. The laser spectrum of fig. $4 \mathrm{~b}$ is taken at an intensity level where stimulated Stokes light is procuded with an energy conversion of $\eta_{\mathrm{S}} \simeq 10^{-2}$. The stimulated Stokes spectrum (see fig. 4c) is broader than the incoming laser spectrum. This fact is due to the shortening of the Stokes pulse during the stimulated scattering process. Of special interest here is the frequency 


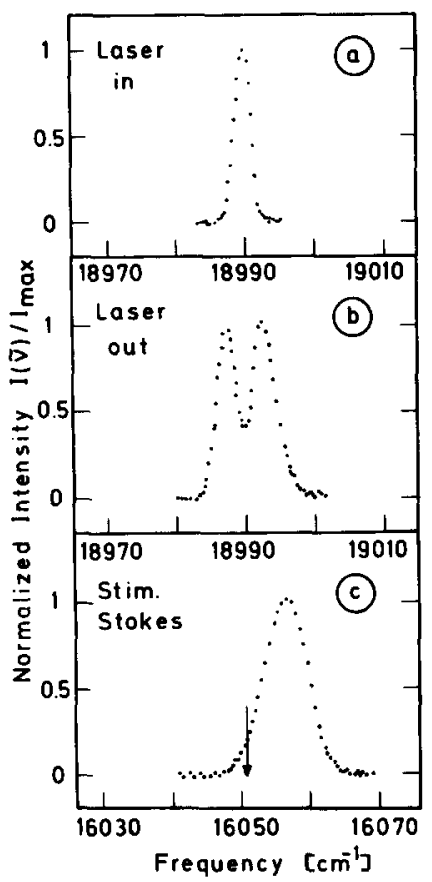

Fig. 4. Experimental results of transient stimulated Raman scattering in liquid $\mathrm{CH}_{3} \mathrm{CCl}_{3}$. (a) Spectrum of the incoming bandwidth limited laser pulse centered at $\tilde{\nu}_{\mathrm{L}} \simeq 18990 \mathrm{~cm}^{-1}$. (b) Spectrum of the laser pulse transmitted through the Raman cell when Stokes light with an energy conversion of $1 \%$ was generated. The spectrum is centered around $\tilde{\nu}_{L}$ with a structure typical for self-phase modulation. (c) Spectrum of the generated stimulated Stokes pulse. The maximum of the stimulated Stokes spectrum is shifted to higher frequencies. The position of spontaneous Raman scattering at $16051 \mathrm{~cm}^{-1}$ is indicated by the arrow.

position of the center of the Stokes pulse. The stimulated Stokes spectrum does not appear at the position of the spontaneous Raman spectrum $\tilde{\nu}_{\mathrm{Sp}}=16051$ $\mathrm{cm}^{-1}$, indicated by the arrow. The stimulated Stokes spectrum is shifted by nearly $6 \mathrm{~cm}^{-1}$ to higher frequencies. More accurately, a frequency difference between the spontaneous and stimulated position of $5.5 \mathrm{~cm}^{-1} \pm 0.5 \mathrm{~cm}^{-1}$ was found at an average value over 20 measurements. This number is in good agreement with the value $\Delta \widetilde{\nu}_{\mathrm{S}}=5.7 \mathrm{~cm}^{-1}$ calculated from eq. (8) using the material parameters of $\mathrm{CCl}_{3} \mathrm{CH}_{3}$ and the experimental data $: n_{2}=1.6 \times 10^{-13} \mathrm{esu} ; \widetilde{\nu}_{\mathrm{L}}=$ $18990 \mathrm{~cm}^{-1}, G=75 ; t_{\mathrm{p}}=7 \mathrm{ps}$, and $g=8 \times 10^{-10}$ $\mathrm{cm} / \mathrm{W}[11]$. As predicted from eq. (8), the frequency shift of the Stokes pulse remains unchanged when the product $I_{\mathrm{L}} l$ is held constant. Varying the cell length between $5 \mathrm{~cm}$ and $1 \mathrm{~cm}$ with a proportional adjustment in laser intensity gave the same experimental Stokes shift.

We have seen shifts of the Stokes spectrum of several wave numbers in other substances with similarly small values of $n_{2}$ (e.g. in $\mathrm{CCl}_{4}$ ). Considerable larger effects are expected in liquids were orientational contributions give much larger nonlinear coefficients $n_{2}$. For instance, $n_{2}$ values of $12.5 \times 10^{-13}$ esu or $120 \times$ $10^{-13}$ esu are reported in the literature for benzene or $\mathrm{CS}_{2}$, respectively [12]. The theory is more complicated in this case since the transient nature of the orientational contributions has to be incorporated into the calculations.

\section{References}

[1] G. Eckhardt, R.W. Hellwarth, F.J. McClung, S.E. Schwarz, D. Weiner and E.J. Woodbury, Phys. Rev. Lett. 9 (1962) 455;

M.D. Martin and E.L. Thomas, IEEE J. Quant. Electron. QE-2 (1966) 196.

[2] J.J. Barrett and M.C. Tobin, J. Opt. Soc. Amer. 56 (1966) 129.

J.R. Maple and J.T. Knudtson, Chem. Phys. Lett. 56 (1978) 241.

[3] A. Penzkofer, A. Laubereau and W. Kaiser, Prog. Quant. Electron. 6 (1979) 55.

[4] J.A. Giordmaine and W. Kaiser, Phys. Rev. 144 (1966) 676.

[5] A. Laubereau and W. Kaiser, Rev. Mod. Phys. 50 (1978) 607.

[6] R.W. Terhune and P.D. Maker, in: Lasers, Vol. 2, ed. A.K. Levine (Marcel Dekker, New York, 1968) p. 295.

[7] A. Laubereau and D. von der Linde, Zeitschr. f. Naturf. 25a (1970) 1626.

[8] A.S. Akhmanov, Mater. Res. Bull. 4 (1969) 455; R.L. Carman, F. Shimizu, C.S. Wang and N. Bloembergen, Phys. Rev. A2 (1970) 60 .

[9] S.A. Akhmanov, M.A. Bol'shov, K.N. Drabovich and A.P. Sukhorukov, JETP Lett. 12 (1970) 388; S.A. Akhmarov, K.N. Drabovich, A.P. Sukhorukov and A.K. Shchednova, Sov. Phys. JETP 35 (1972) 279.

[10] W. Zinth, A. Laubereau and W. Kaiser, Optics. Comm. 22 (19.77) 161.

[11] M.J. Colles and J.E. Griffiths, J. Chem. Phys. 56 (1972) 3384;

$\boldsymbol{n}_{2}$ was calculated from: D. Milam, M.J. Wever and A.J. Glass, Appl. Phys. Lett. 31 (1977) 822.

[12] M. Paillette, Ann. Phys. (Paris) 4 (1969) 671. 